\title{
A Result Analysis of Weighted Guided Image Filtering
}

\author{
Rohit Sharma ${ }^{1}$, Harsh Lohiya ${ }^{2}$, Kailash Patidar ${ }^{3}$ \\ M. Tech Scholar, Department of CS, SSSIST, Bhopal, India ${ }^{1}$ \\ Asst. Prof., Department of CS, SSSIST, Bhopal, India ${ }^{2}$ \\ H.O.D., Department of CS, SSSIST, Bhopal, India ${ }^{3}$
}

\begin{abstract}
A result analysis of Weighted Guided Image Filtering, present days various image filtering method in computer vision and image dispensation that are helpful for some variety of noise, but they invariably put together certain supposition concerning the properties of the signal and/or noise to lack the generalization for different image noise reduction. This study explains an absolutely unique generalized guided image filtering methodology by means of the reference image create by signal sub-space projection (SSP) method. It adopts advanced parallel investigation with Monte Carlo simulations to pick out the dimensionality of signal subspace contained by the patch-based noisy images. The noiseless image is rebuilt as of the noisy image estimated onto the many Eigen images by element analysis. Training/test image is utilized to work out the relationship between the best possible parameter value and noise divergence that maximizes the output peak signal/noise ratio (PSNR). The best possible consideration of the estimated algorithmic rule are often by design chosen by means of noise deviation estimation based on the smallest singular value of the patch-based image by singular value decomposition (SVD). Lastly, we have a tendency to present a quantitative and qualitative assessment of the projected algorithmic rule with the traditional guided filter and different state-of-theart methods with respect to the choice of the image patch and neighbourhood window sizes.
\end{abstract}

Keywords: Edge-preserving smoothing, weighted guided image filter, edge-aware weighting, detail enhancement, haze removal, exposure fusion.

\section{INTRODUCTION}

Many applications in computer vision and computer graphics engages image filtering to restrain and/or remove content in pictures. Many outside computer vision purpose like video surveillance object recognition, visual perception, tracking, intelligent vehicles and remote sensing arrangement etc., suppose that the input images have clear visibility. Unfortunately, this can be not forever true in many situations, particularly, haze and fog weather occurring more and more frequently. Outside pictures or videos are typically degraded by light scattering and absorbing from the aerosols, like dust, mist, and fumes within the atmosphere, here regarded as haze. The captured scenes suffer from poor visibility, contrast, brightness, luminousness and hazy color.

With the aid of atmospherically optics theories, one can justify the effects that haze has on the visibility of a scene and eventually of a picture taken of that scene. Moreover, with the development of computer graphics technology, it's possible to enhance the visibility in terms of range, color verisimilitude and feature separation in digital images. In this the term "dehazing" stand for that to provide a picture of a scene that doesn't contain hazes effects though the source of that image originally comprised haze. A multiple ways that to accumulate digital pictures from the important world: digital cameras, scanners, CT, and resonance imaging to name a few. In each case what humans see are pictures. However, once transforming this to our digital devices what we tend to record are numerical values for every of the points of the image [19]. The widespread accessibility of relatively low-priced personal computers has heralded a revolution in digital image process activities among scientists and also the consumer population generally.

In digital image, a pixel may be a physical purpose during a formation image, or the smallest addressable component in an all points available display device; therefore it's the smallest controllable component of an image depicted on the screen. The address of a picture element corresponds to its physical coordinates. LCD pixels are manufactured during a two-dimensional grid, and are usually depicted using dots or squares; however CRT pixels correspond to their timing mechanisms and sweep rates. Every pixel may be a sample of an original image; a lot of samples usually provide a lot of correct representations of the initial. A grayscale or grayscale digital image is a picture during which the value of every pixel may be a single sample, that is, it carries only intensity info. Pictures of this kind, additionally called blackand-white, are composed completely of shades of gray, varied from black at the weakest intensity to white at the strongest. 


\section{THEORY}

\section{A. Edge Detection}

Edge detection is that the name for a collection of mathematical methods that aim at identifying points during a digital image at that the image brightness changes sharply or, additional formally, has discontinuities. The points at that image brightness changes sharply are usually organized into a set of curved line segments termed edges. a similar drawback of finding discontinuities in 1D signal is known as step detection and also the drawback of finding signal discontinuities over time is understood as modification detection. Edge detection may be a basic tool in image processing, machine vision and pc vision, significantly within the areas of feature detection and have extraction.

The purpose of detection sharp changes in image brightness is to capture vital events and changes in properties of the world. In the ideal case, the results of applying an edge detector to a picture could result in a set of connected curves that indicate the boundaries of objects, the boundaries of surface markings also as curves that correspond to discontinuities in surface orientation. Thus, applying an edge detection algorithmic rule to a picture could significantly reduce the number of \{information\} to be processed and will so filter out information that will be considered less relevant, whereas conserving the necessary structural properties of a picture? If the edge detection step is successful, the following task of decoding the data contents within the original image could so be well simplified. However, it's not always possible to get such ideal edges from reality pictures ofmoderatecomplexity.Edgesextractedfromnontrivialimagesareoftenhamperedby fragmentation that means that the edge curves are not connected, missing edge segments also as false edges not similar to interesting phenomena within the image therefore complicating the following task of decoding the image information. Edge detection is one in all the basic steps in image processing, image analysis, image pattern recognition, and pc vision techniques.

The edges extracted from a two-dimensional image of a three-dimensional scene will be classier as either viewpoint dependent or viewpoint independent. A viewpoint independent edge usually reflects inherent properties of the threedimensional objects, like surface markings and surface form. A viewpoint dependent edge could modification because the viewpoint changes, and usually reflects the geometry of the scene, like objects occluding each other.

A typical edge may for example be the border between a block of red color and a block of yellow. In distinction a line (as will be extracted by a ridge detector) will be a small range of pixels of a different color on an otherwise unchanging background. For a line, there might so usually be one edge on both sides of the line.

\section{B. Canny Edge Detection}

Title John canny thought of the mathematical drawback of derivation an optimal smoothing filter given the standards of detection, localization and minimizing multiple responses to one edge.[11] He showed that the optimal filter given these assumptions may be a total of 4 exponential terms. He additionally showed that this filter may be well approximated by first-order derivatives of Gaussians. Canny additionally introduced the notion of non-maximum suppression, which suggests that given the presmoothing filters, edge points are defined as points wherever the gradient magnitude assumes a local most within the gradient direction. Searching for the zero crossing of the 2nd derivative on the gradient direction was first planned by Haralick.[12]

Unless the preconditions are significantly appropriate, it's hard to find an edge detector that performs significantly higher than the canny edge detector. The canny-Deriche detector was derived from similar mathematical criteria because the Canny edge detector, though starting from a discrete viewpoint and so leading to a collection of algorithmic filters for image smoothing rather than exponential filters or Gaussian filters.

\section{METHOD}

Edge-Preserving Decomposition-based method is introduced to estimate transmission map for a haze image so as to design a single image haze removal algorithm from the Koschmiedars law without using any prior. In particular, weighted guided image filter is adopted to decompose simplified dark channel of the haze image into a base layer and a detail layer [18]. The transmission map is estimated from the base layer, and it is applied to restore the haze-free image.

$$
\tilde{t}(X)=1-\omega \frac{M \operatorname{ean}\left(d^{I}(X)\right)}{A_{0}}
$$

When $\omega$ is large, that is the image is very hazy.

$$
t(x)=\max \left(\tilde{t}(x), 1-\frac{d^{I}(x)}{A_{0}}\right)
$$




$$
J(x)=\frac{I(x)-A}{\max \left(t(x), t_{0}\right)}+A
$$

Algorithm to find the haze free image is as follows:

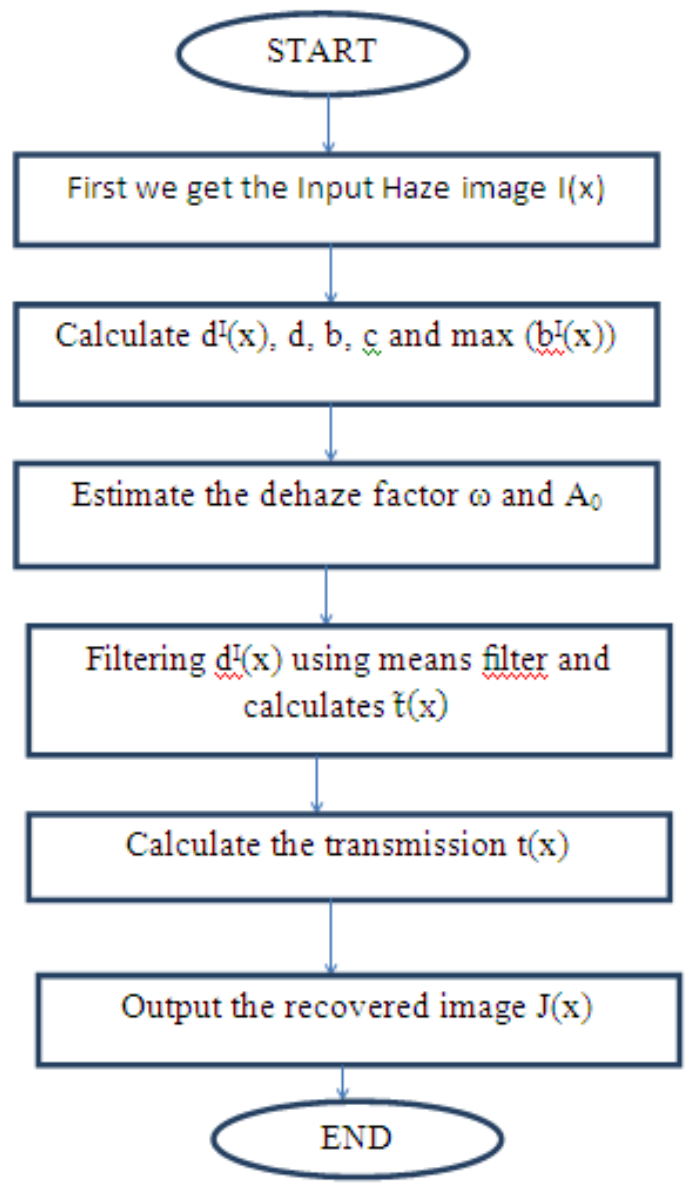

Fig.1 data flow diagram

\section{IV.RESULT}

In the analysis of edge preserving of image we can see that image with the haze is analyzed and gives the following results.

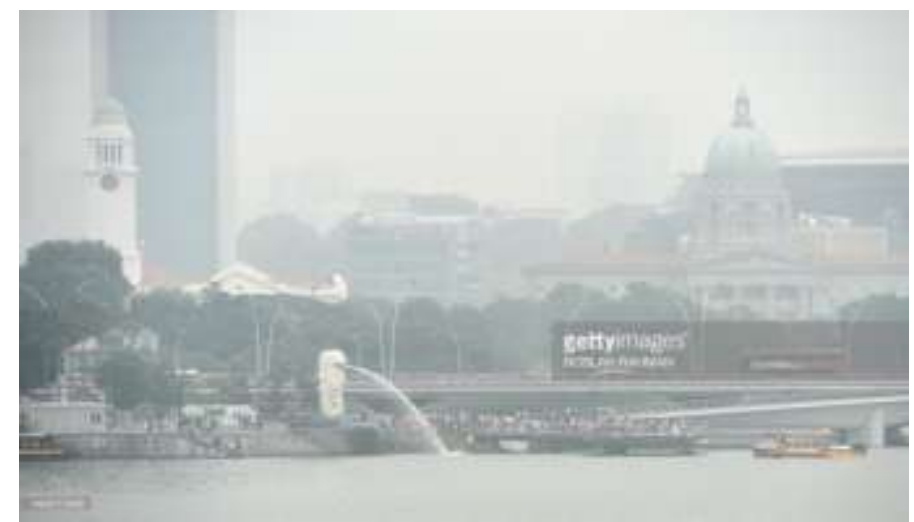

Fig. 2 Input images with Haze

In the above figure we can see the input image taken which contains haze and we analysis the different technique to: 
International Advanced Research Journal in Science, Engineering and Technology ISO 3297:2007 Certified

Vol. 4, Issue 5, May 2017

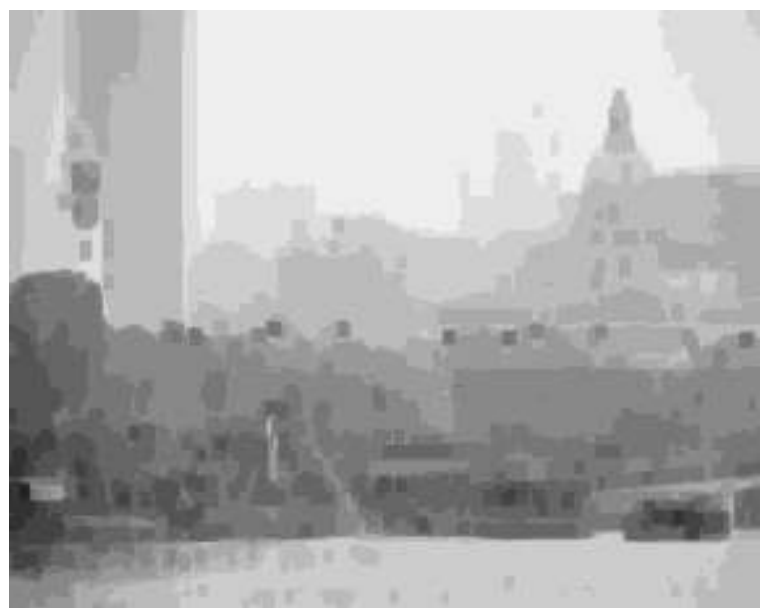

Fig. 3 Result image in dark form after haze removal

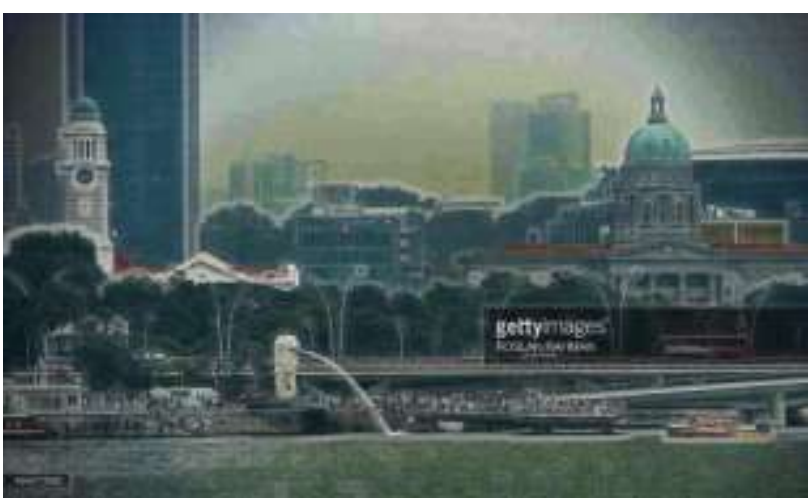

Fig. 4 Radiant image after haze removal

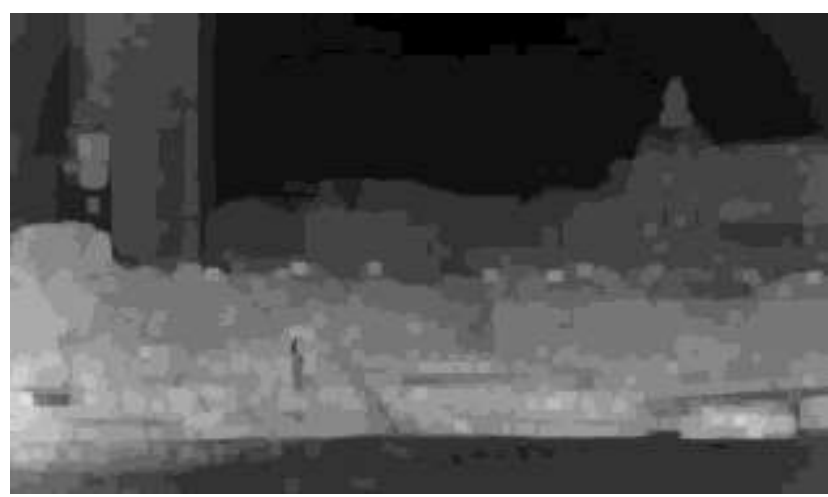

Fig. 5 transient image after haze removal

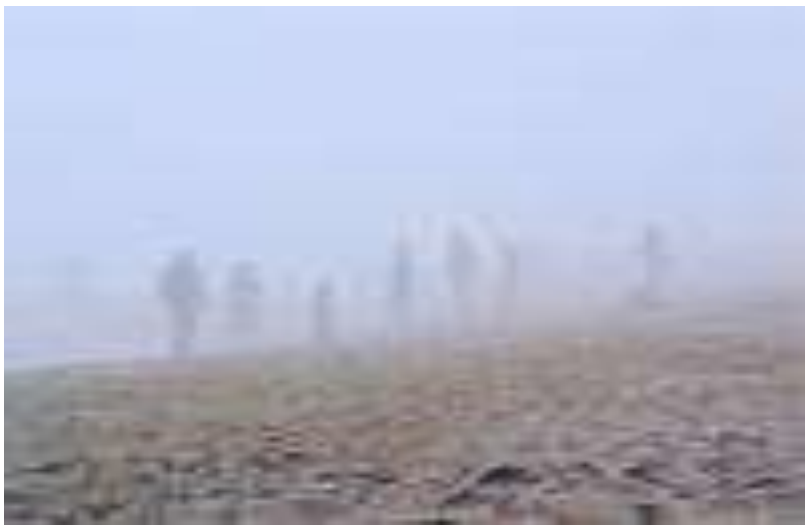

Fig. 6 another input image 


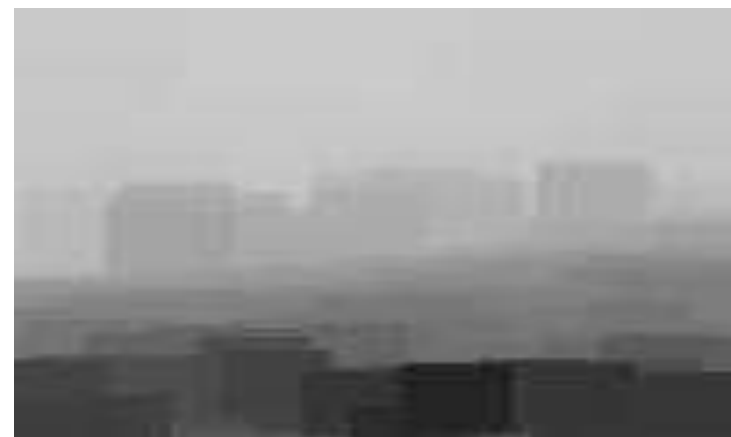

Fig.7 Result image in dark form after haze removal

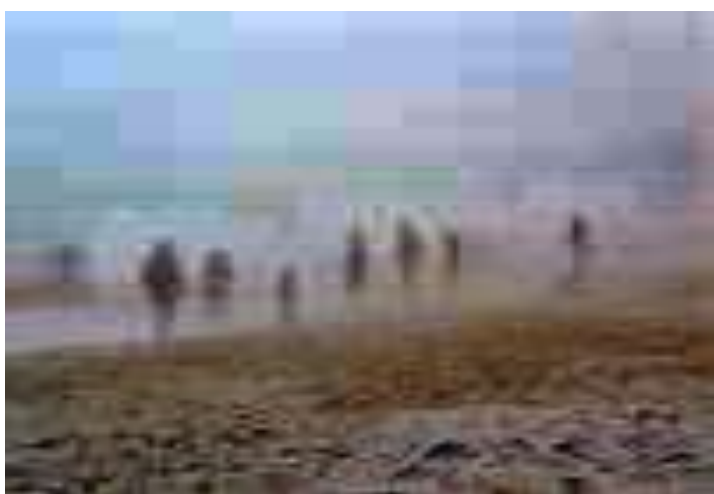

Fig.8 Radiant image after haze removal

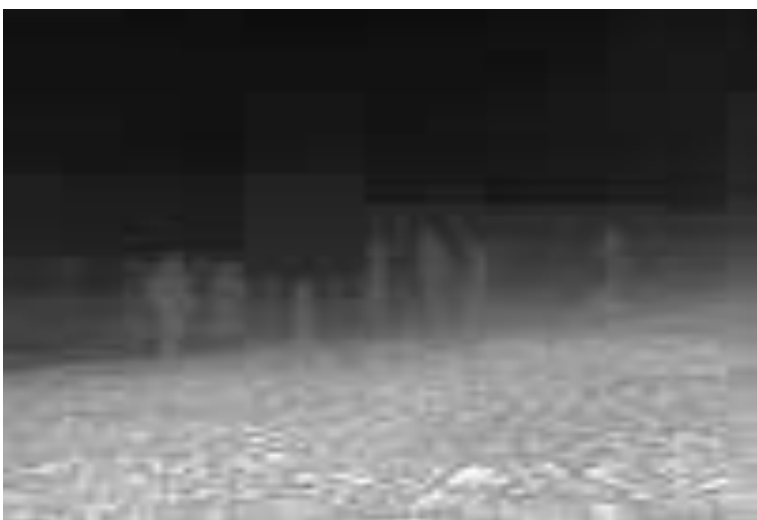

Fig. 9 Transient image after haze removal

\section{CONCLUSION}

Even A weighted guided image filter (WGIF) is proposed in this paper by incorporating an edge-aware weighting into the guided image filter (GIF). The WGIF conserve prickly edges as well as existing global filters, and the complexity of the WGIF is $\mathrm{O}(\mathrm{N})$ for an picture with $\mathrm{N}$ pixels which is almost the same as the GIF. Due to the simplicity of the WGIF, it has numerous applications in the field of computational photography and image processing. Particularly, it is applied to learn particular image specification enhancement, single picture haze removal, and fusion of differently exposed images. Experimental consequences show that the resultant algorithms can generate images with excellent visual quality as those of global filters, and at the equivalent time the operation times of the projected algorithms are comparable to the GIF base algorithms

\section{REFERENCES}

[1] S. Zhengguo Li, Jinghong Zheng, Zijian Zhu, Wei Yao, Shiqian Wu "Weighted Guided Image Filtering" IEEE Transactions on Image Processing, Vol. 24, No. 1, January 2015 1057-7149 @ 2014 IEEE.

[2] Kaiming He, Jian Sun, Xiaoou Tang "Guided Image Filtering" IEEE TRANSACTIONS ON PATTERN ANALYSIS AND MACHINE INTELLIGENCE, VOL. 35,2013 0162-8828/13/\$31.00 2013 IEEE 
[3] Pierre Charbonnier, Laure Blanc-F'eraud, Gilles Aubert, Michel Barlaud "Deterministic Edge-Preserving Regularization in Computed Imaging” IEEE TRANSACTIONS ON IMAGE PROCESSING, VOL. 6, NO. 2, FEBRUARY 1997

[4] T. Mertens, J. Kautz, and F. Van Reeth, "Exposure fusion: A simple and practical alternative to high dynamic range photography," Comput. Graph. Forum, vol. 28, no. 1, pp. 161-171, 2009.

[5] K. Moorthy and A. C. Bovik, “A two-step framework for constructing blind image quality indices,” IEEE Signal Process. Lett., vol. 17, no. 5, pp. 513-516, May 2010.

[6] L. I. Rudin, S. Osher, and E. Fatemi, "Nonlinear total variation based noise removal algorithms," Phys. D, Nonlinear Phenomena, vol. 60, nos. 1-4, pp. 259-268, Nov. 1992.

[7] Z. G. Li, J. H. Zheng, and S. Rahardja, "Detail-enhanced exposure fusion,” IEEE Trans. Image Process., vol. 21, no. 11, pp. 4672-4676, Nov. 2012.

[8] R. Fattal, M. Agrawala, and S. Rusinkiewicz, "Multiscale shape and detail enhancement from multi-light image collections," ACM Trans. Graph, vol. 26, no. 3, pp. 51:1-51:10, Aug. 2007.

[9] R. C. Gonzalez and R. E. Woods, Digital Image Processing. Upper Saddle River, NJ, USA: Prentice-Hall, 2002.

[10] Levin, D. Lischinski, and Y. Weiss, “A closed-form solution to natural image matting," IEEE Trans. Pattern Anal. Mach. Intell., vol. 30, no. 2, pp. 228-242, Feb. 2008.

[11] J. Preetham, P. Shirley, and B. Smits, “A practical analytic model for daylight,” in Proc. SIGGRAPH, 1999, pp. 91-100.

[12] S. G. Narasimhan and S. K. Nayar, "Chromatic framework for vision in bad weather," in Proc. IEEE Conf. Comput. Vis. Pattern Recognit. (CVPR), Jun. 2000, pp. 598-605.

[13] Shih-Chia Huang, Jian-Hui Ye, and Bo-Hao Chen, "An Advanced Single-Image Visibility Restoration Algorithm for Real-World Hazy Scenes", IEEE transaction on industrial engineering VOL. 62, NO. 5, MAY 2015

[14] Zhengguo Li, and JinghongZheng, "Edge-Preserving Decomposition-Based Single Image Haze Removal", IEEE Transaction on image processing, VOL. 24, NO. 12, DECEMBER 2015

[15] Jin-Hwan Kim, Jae-Young Sim, and Chang-Su Kim "Single Image Dehazing Based On Contrast Enhancement" 978-1 -4577-0539-2011 IEEE

[16] Bin Xie, Fan Guo, Zixing Cai "Improved Single Image Dehazing Using Dark Channel Prior and Multi-Scale Retinex" 2010 International Conference on Intelligent System Design and Engineering Application

[17] Qingsong Zhu, Jiaming Mai, Ling Shao “A Fast Single Image Haze Removal Algorithm Using Color Attenuation Prior” IEEE transactions on image processing, vol. 24, no. 11, november 2015

[18] Hulbert E. O. Optics at atmospheric haze. J. Opt. Soc. Am., 31:467-476, 1941. 